\title{
Modelling of Anisotropic Viscoelastic Behaviour in Super-Pressure Balloons
}

\author{
T. Gerngross* and S. Pellegrino ${ }^{\dagger}$ \\ University of Cambridge, Cambridge, CB2 1PZ, UK
}

\begin{abstract}
Large balloon structures are made of thin anisotropic polymeric film that shows considerable time-dependent material behaviour. While the material has been fully characterised and a detailed time-dependent material description by means of a nonlinear viscoelastic Schapery material model is available, there is no suitable numerical implementation for finite element analysis. This paper presents an algorithm for nonlinear viscoelastic and anisotropic material behaviour. After an overview of the material model, a detailed description of the iterative algorithm is given including the full set of equations. The model is implemented by means of a user-defined subroutine in ABAQUS. For verification a set of cylindrical balloons is observed analytically and experimentally and the results are compared.
\end{abstract}

\section{Nomenclature}

$\Delta t$

$t-\Delta t$

$T$

$\varepsilon$

$\Delta \varepsilon$

$\lambda_{n}$

$\sigma$

$\Delta \sigma$

$\bar{\sigma}_{\text {eff }}$

$\sigma_{\text {axial }}$

$\sigma_{\text {hoop }}$

$\sigma_{M D}$

$\sigma_{T D}$

$\tau$ coefficients for effective stress

temperature-dependent shift

stress-dependent shift

transient compliance

elastic compliance

$\mathrm{n}^{\text {th }}$ coefficient of a Prony series

variable summarising parts of an equation

variable summarising parts of an equation

force

nonlinear change of elastic compliance

nonlinear change of transient compliance

nonlinear sensitivity of transient stress

pressure

heredity integral for stress history

radius

strain residual

coefficient relating machine and transverse direction

current time

time increment

previous time

thickness

total strain

strain increment

$\mathrm{n}^{\text {th }}$ inverse of relaxation time

total stress

stress increment

effective stress

stress parallel to the cylinder axis

stress in circumferential direction

stress in machine direction

stress in transverse direction

variable for values of time between 0 and $\mathrm{t}$

* Research student, Department of Engineering, Trumpington Street.

$\dagger$ Professor of Structural Engineering, Department of Engineering, Trumpington Street. Associate Fellow AIAA. 


\section{Introduction}

State-of-the-art large super-pressure stratospheric balloons make use of thin polymeric films to form a sealed envelope that is contained by a series of meridional tendons. This concept originates from parachute design and was first realized (with different materials) by J. Nott in 1984, Figure 1 . In the balloon the polymeric film is subject to a biaxial state of stress whose details depend on the cutting pattern, stiffness of the film vs. stiffness of the tendons, etc. Viscoelastic effects, which are usually significant in the film, play a significant role in the stress distribution and shape of these balloons.

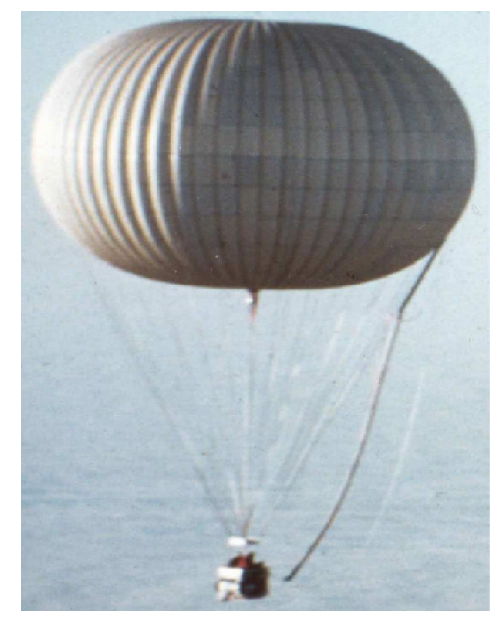

Figure 1. Endeavour super-pressure balloon, courtesy of Julian Nott.

So far pseudo-elastic material properties have been generally assumed for the design of the balloon structure. However, following a number of anomalies during flight tests of NASA Ultra-Long-DurationBalloons (ULDB) ${ }^{1}$ it has been realized that the behaviour of super-pressure balloons is much more complex than assumed at first. As the complexity of these balloons is better grasped, ${ }^{3,4}$ detailed experimental validation of the analysis models is being initiated, and this in turn requires that details of the time-dependent material behavior be also included in the models.

This paper is part of an ongoing effort to develop more realistic models for super-pressure balloons, validated with reference to fully representative physical models. Here we present a nonlinear, anisotropic, viscoelastic material model implemented through a user-defined material subroutine in the ABAQUS finite-element package. Based on this approach, we have predicted the creep strains in a cylindrical structure and compared them with actual measurements.

\section{Balloon Material}

A linear low density polyethylene (LLDPE) film, called StratoFilm 372, has been used for many years for NASA balloons. This film was produced as a monolayer extrusion with a blow-up-ratio (BUR) of three, and a thickness of $0.02 \mathrm{~mm}$ was achieved. This material has been fully characterised, resulting in a detailed time-dependent material description. ${ }^{5-7}$

For the ULDB a new material, called StratoFilm 430, has been introduced. This consists of a three layer co-extrusion using the same LLDPE resin as for SF372. A different die and a reduced BUR have resulted in a film with a thickness of $0.038 \mathrm{~mm}$. It has been suggested that these changes affect only the transverse direction properties of the film and so that the properties in the machine direction remain unchanged. ${ }^{6}$

\section{Nonlinear Viscoelastic Models}

A general introduction to the field of nonlinear viscoelasticity is provided in textbooks. ${ }^{8,9}$ In reference 2 we have presented an attempt to model the time-dependent material behavior of LLDPE using the creep/relaxation models available in ABAQUS. These were compared to a viscoelastic model that uses 
the ABAQUS built-in interface for user-defined subroutines. This alternative approach was found to be much more accurate, and hence the following sections describe how the user defined material (UMAT) has been implemented and how it has been verified with biaxial experimental data from cylinder tests.

\section{A. Schapery Uniaxial Constitutive Equation}

Schapery ${ }^{10}$ derived a nonlinear viscoelastic constitutive material model based on the thermodynamics of irreversible processes, where the transient material behavior is defined by a master creep function. Nonlinearities can be considered by including factors that are functions of stress and temperature. Further, horizontal shift factors enable coverage of wide temperature/stress ranges:

$$
\varepsilon^{t}=g_{0}^{t} D_{0} \sigma^{t}+g_{1}^{t} \int_{0}^{t} \Delta D^{\left(\psi^{t}-\psi^{\tau}\right)} \frac{d\left(g_{2}^{\tau} \sigma^{\tau}\right)}{d \tau} d \tau
$$

where the reduced time is

$$
\psi^{t}=\int_{0}^{t} \frac{d \tau}{a_{\sigma}(T, \sigma) a_{T}(T)}
$$

The first term in Equation (1) represents the elastic response of the material, provided by the instantaneous elastic compliance $D_{0}$, while the second term describes the transient response, defined by the transient compliance function, $\Delta D$. The other parameters are the horizontal shift factors for the master-curve.

\section{B. Multiaxial Model Including Anisotropy}

A general multiaxial formulation of Equation (1) has been given by Schapery ${ }^{10}$ with the nonlinear function being an arbitrary function of stress. Schapery found that the Poisson's ratio has only a weak time-dependence and hence a single time-dependent function is sufficient to characterize all elements of the linear viscoelastic creep compliance matrix. ${ }^{11}$

Rand and co-workers ${ }^{6,12}$ further simplified this relationship by assuming that the time-dependence in any material direction is linearly related to that observed in the machine direction:

$$
\Delta D_{i j}=S_{i j} \Delta D
$$

This can be similarly done for the instantaneous elastic compliance, and so Equation (1) can be rewritten as:

$$
\varepsilon_{i}^{t}=g_{0}^{t} S_{i j}^{0} D_{0} \sigma_{j}^{t}+g_{1}^{t} \int_{0}^{t} S_{i j} \Delta D^{\left(\psi^{t}-\psi^{\tau}\right)} \frac{d\left(g_{2}^{\tau} \sigma_{j}^{\tau}\right)}{d \tau} d \tau
$$

Note that in this model there is no decomposition into deviatoric and volumetric stress/strain. However, if shear plays a pronounced role in a particular material, this can be accounted for by adjusting the corresponding factors of $S_{i j}$.

Since the material response in any direction is based on the properties in the machine direction, one assumes $S_{11}^{0}=1$ and $S_{11}=1$. Anisotropic behaviour is accounted for by setting the remaining coefficients $S_{i i}^{0}$ and $S_{i i}$ to values different from one.

The nonlinearity functions were found by Schapery to be scalar functions of a single effective stress $\bar{\sigma}_{\text {eff }}$, which was expressed in terms of the octahedral shear stress. In the special case of thin films, a biaxial state of stress is of course assumed and so the effective stress can be written in the form:

$$
\bar{\sigma}_{e f f}^{t}=\sqrt{\sigma_{1}^{t, 2}+2 A_{12} \sigma_{2}^{t} \sigma_{3}^{t}+A_{22} \sigma_{3}^{t, 2}+A_{66} \tau_{12}^{t, 2}}
$$

In the case of a uniaxial test in the machine direction the effective stress reduces to $\bar{\sigma}_{e f f}=\sigma_{1}$. Similarly $A_{22}$ can be found from uniaxial tests in the transverse direction.

\section{Viscoelastic Model for ULDB Balloon Film}

As mentioned in Section II the StratoFilm 372 has been extensively studied. ${ }^{5-7}$ After some initial creep tests we decided to use Rand's master curve and nonlinearity functions for SF372.

The plots in Figures 2 and 3 show the master curve and the nonlinearity functions, from references 5 and 7. These curves relate to a temperature of $23^{\circ} \mathrm{C}$ and to an instantaneous elastic compliance of $D_{0}=3 \cdot 10^{-10} \frac{1}{P a}$. The nonlinearity parameters $g_{0}$ and $g_{1}$ are equal to one. The multiaxial parameters $S_{i j}^{0}$ and $S_{i j}$ are assumed to be the same for the two films. They were determined in reference 6 for a biaxial state of stress at $23^{\circ} \mathrm{C}$; their values are listed in Table 1 . 


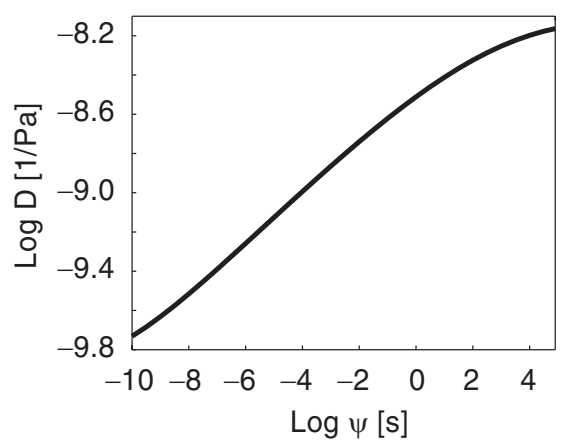

Figure 2. Master curve for transient creep compliance of SF372 in machine direction, from reference 7 .
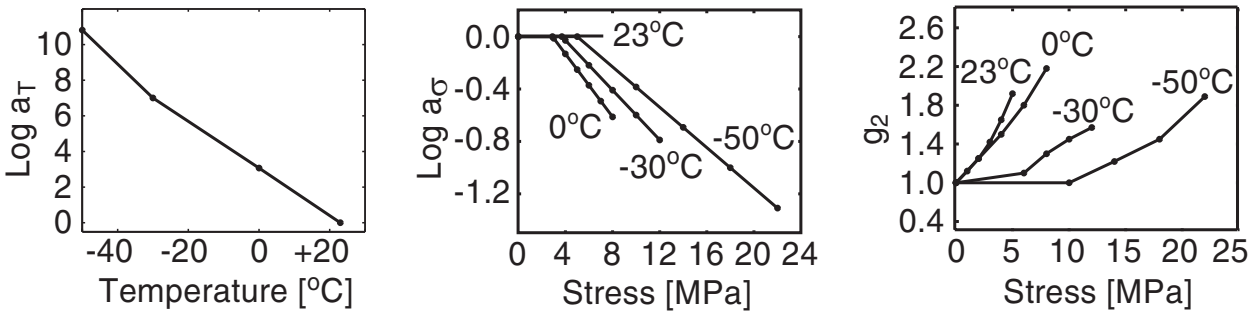

Figure 3. Nonlinearity functions for SF372, from reference 7.

\section{ABAQUS User Defined Subroutine}

\section{A. Methodology}

To use Schapery's single-integral constitutive model in a numerical algorithm, Equation (4) needs to be rewritten in incremental form. A numerical integration method was presented by Haj-Ali and Muliana ${ }^{13}$ for a three-dimensional, isotropic material. Based on the integration method proposed in reference 13, an algorithm has been developed for anisotropic material behavior that implements the biaxial approach of Rand and co-workers. ${ }^{6}, 12$ This algorithm was implemented in ABAQUS, but would be equally suitable for any displacement based finite element software, where strain components are used as the independent state variables.

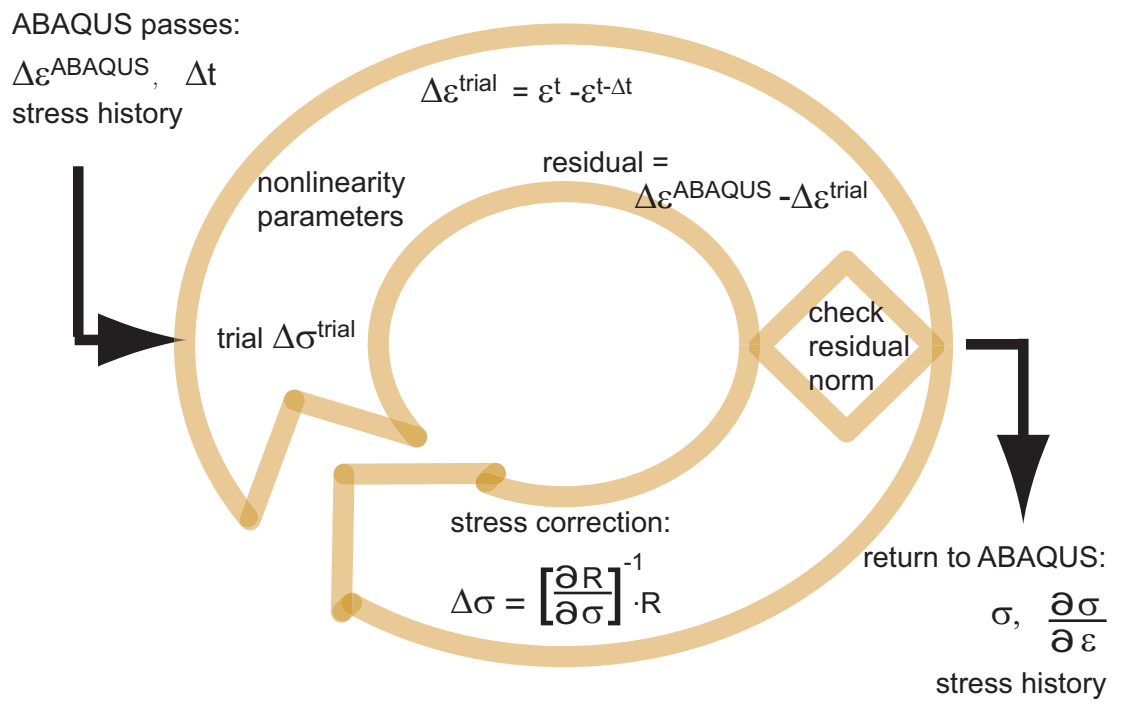

Figure 4. Iterative algorithm in UMAT.

The ABAQUS interface for a user-defined material (UMAT) passes the current time increment $\Delta t$ and the corresponding strain increment $\Delta \varepsilon$, determined using the Jacobian matrix at the end of the 


\begin{tabular}{|c|c|c|c|c|c|}
\hline$S_{12}$ & $S_{22}$ & $S_{66}$ & $A_{12}$ & $A_{22}$ & $A_{66}$ \\
\hline-0.49 & 0.72 & 3.60 & -1.09 & 1.18 & 6.05 \\
\hline
\end{tabular}

Table 1. Coefficients for SF372 at $23^{\circ} \mathrm{C}$

previous time increment. In turn, it requires at the end of the current time increment an update of the stresses $\sigma_{j}^{t}$ and the Jacobian matrix $\frac{\partial \sigma_{j}^{t}}{\partial \varepsilon_{i}^{t}}$.

The incremental method requires the transient strain function $\Delta D$ to be expressed in terms of a sum of exponentials, called a Prony series, and the strain/stress history needs to be stored at the end of each increment for each strain/stress component and each Prony term.

Every time UMAT is called, it starts with an estimation of a trial stress increment $\Delta \sigma^{t, t r i a l}$ based on the nonlinearity parameters at the end of the previous time increment. With this initial guess an iterative loop is entered, where the integration in Equation (4) yields $\Delta \varepsilon^{t, t r i a l}$, which is compared with $\triangle \varepsilon^{t, A B A Q U S}$. If required, the stresses and the nonlinearity parameters are corrected and the loop is repeated. Alternatively, if the strain error residual is below a specified tolerance (set to $t o l=10^{-7}$ ) UMAT exits the loop and updates the Jacobian matrix and the stresses.

The iterative algorithm is depicted in Figure 4 and the detailed derivation of the equations is given in the next section.

\section{B. Iterative Algorithm}

In this section the equations required for the iterative, anisotropic, multiaxial formulation of the algorithm are outlined.

The current total strain in terms of the heredity integral formulation can be written as:

$$
\begin{aligned}
\varepsilon_{i}^{t} & =\left\{g_{0}^{t} S_{i j}^{0} D_{0}+g_{1}^{t} g_{2}^{t} S_{i j} \sum_{n=1}^{N} D_{n}\left[1-\frac{1-\exp \left(-\lambda_{n} \Delta \psi^{t}\right)}{-\lambda_{n} \Delta \psi^{t}}\right]\right\} \sigma_{j}^{t} \\
& -g_{1}^{t} S_{i j} \sum_{n=1}^{N} D_{n}\left[\exp \left(-\lambda_{n} \Delta \psi^{t}\right) q_{j, n}^{t-\Delta t}-g_{2}^{t-\Delta t} \sigma_{j}^{t-\Delta t} \frac{1-\exp \left(-\lambda_{n} \Delta \psi^{t}\right)}{-\lambda_{n} \Delta \psi^{t}}\right] \\
& =\bar{D}_{i j}^{t} \sigma_{j}^{t}-f_{i}^{t}
\end{aligned}
$$

which define the heredity integral $q_{j, n}^{t-\Delta t}$ at the end of previous time $t-\Delta t$. At the end of each time increment this has to be updated and stored for every Prony series term and every strain component, using

$$
q_{j, n}^{t}=\exp \left(-\lambda_{n} \Delta \psi^{t}\right) q_{j, n}^{t-\Delta t}+\frac{1-\exp \left(-\lambda_{n} \Delta \psi^{t}\right)}{-\lambda_{n} \Delta \psi^{t}}\left(g_{2}^{t} \sigma_{j}^{t}-g_{2}^{t-\Delta t} \sigma_{j}^{t-\Delta t}\right)
$$

The strain increment can be written as

$$
\begin{aligned}
\Delta \varepsilon_{i}^{t} & =\bar{D}_{i j}^{t} \sigma_{j}^{t}-\bar{D}_{i j}^{t-\Delta t} \sigma_{j}^{t-\Delta t} \\
& -\sum_{n=1}^{N} D_{n}\left[g_{1}^{t} \exp \left(-\lambda_{n} \Delta \psi^{t}\right)-g_{1}^{t-\Delta t}\right] S_{i j} q_{j, n}^{t-\Delta t} \\
& -g_{2}^{t-\Delta t} \sum_{n=1}^{N} D_{n}\left[g_{1}^{t-\Delta t} \frac{1-\exp \left(-\lambda_{n} \Delta \psi^{t-\Delta t}\right)}{-\lambda_{n} \Delta \psi^{t-\Delta t}}-g_{1}^{t} \frac{1-\exp \left(-\lambda_{n} \Delta \psi^{t}\right)}{-\lambda_{n} \Delta \psi^{t}}\right] S_{i j} \sigma_{j}^{t-\Delta t}
\end{aligned}
$$

The initial trial stresses $\sigma_{j}^{t, t r i a l}$ are found by assuming the current nonlinearity parameters $g_{0}, g_{1}$, and $g_{2}$ to be equal to the end of the previous time increment. Also, $\psi^{t}$ is assumed to equal $\psi^{t-\Delta t}$. This enables Equation (8) to be simplified and rewritten in terms of the stress increments:

$$
\begin{aligned}
\Delta \sigma_{j}^{t, \text { trial }} & =\sigma_{j}^{t}-\sigma_{j}^{t-\Delta t} \\
& =\frac{1}{\bar{D}_{i j}^{t, t r i a l}}\left\{\Delta \varepsilon_{i}^{t}+g_{1}^{t, \text { trial }} \sum_{n=1}^{N} D_{n}\left[\exp \left(-\lambda_{n} \Delta \psi^{t}\right)-1\right] S_{i j} q_{j, n}^{t-\Delta t}\right\}
\end{aligned}
$$

where $\bar{D}_{i j}^{t, t r i a l}$ is the same as in Equation (6) but with the approximated nonlinearity factors from the end of the previous time increment. 
Based on the trial stresses $\sigma_{j}^{t, \text { trial }}$ from Equation (9), the trial strain increments $\Delta \varepsilon_{i}^{t, t r i a l}$ at the current time $t$ are calculated. The residual strain resulting from the difference between these trial strain increments and the ABAQUS strain increments $\Delta \varepsilon_{i}^{t, a b a q u s}$ is computed from:

$$
\begin{aligned}
R_{i}^{t} & =\Delta \varepsilon_{i}^{t, \text { trial }}-\Delta \varepsilon_{i}^{t, \text { abaqus }} \\
& =\bar{D}_{i j}^{t} \sigma_{j}^{t}-\bar{D}_{i j}^{t-\Delta t} \sigma_{j}^{t-\Delta t} \\
& -\sum_{n=1}^{N} D_{n}\left[g_{1}^{t} \exp \left(-\lambda_{n} \Delta \psi^{t}\right)-g_{1}^{t-\Delta t}\right] S_{i j} q_{j, n}^{t-\Delta t} \\
& -g_{2}^{t-\Delta t} \sum_{n=1}^{N} D_{n}\left[g_{1}^{t-\Delta t} \frac{1-\exp \left(-\lambda_{n} \Delta \psi^{t-\Delta t}\right)}{-\lambda_{n} \Delta \psi^{t-\Delta t}}-g_{1}^{t} \frac{1-\exp \left(-\lambda_{n} \Delta \psi^{t}\right)}{-\lambda_{n} \Delta \psi^{t}}\right] S_{i j} \sigma_{j}^{t-\Delta t} \\
& -\Delta \varepsilon_{i}^{t, \text { abaqus }}
\end{aligned}
$$

The derivative of the residual can then be written as:

$$
\begin{aligned}
\frac{\partial R_{i}^{t}}{\partial \sigma_{j}^{t}} & =\bar{D}_{i j}^{t} \delta_{i j}+\left\{\frac{\partial \bar{D}_{i j}^{t}}{\partial \bar{\sigma}_{e f f}^{t}} \sigma_{j}^{t}\right. \\
& +\frac{\partial g_{1}^{t}}{\partial \bar{\sigma}_{e f f}^{t}} S_{i j} \sum_{n=1}^{N} D_{n}\left[-\exp \left(-\lambda_{n} \Delta \psi^{t}\right) q_{j, n}^{t-\Delta t}+g_{2}^{t-\Delta t} \frac{1-\exp \left(-\lambda_{n} \Delta \psi^{t}\right)}{-\lambda_{n} \Delta \psi^{t}} \sigma_{j}^{t-\Delta t}\right] \\
& +\frac{\partial a_{\sigma}^{t}}{\partial \bar{\sigma}_{e f f}^{t}} g_{1}^{t} S_{i j} \sum_{n=1}^{N} D_{n}\left[-\frac{\lambda_{n} \Delta \psi^{t}}{a_{\sigma}} \exp \left(-\lambda_{n} \Delta \psi^{t}\right) q_{j, n}^{t-\Delta t}\right. \\
& \left.\left.+\frac{g_{2}^{t-\Delta t}}{a_{\sigma}^{t}}\left(\frac{1-\exp \left(\lambda_{n} \Delta \psi^{t}\right)}{\lambda_{n} \Delta \psi^{t}}-\exp \left(-\lambda_{n} \Delta \psi^{t}\right)\right) \sigma_{j}^{t-\Delta t}\right] \frac{\partial \bar{\sigma}_{e f f}^{t}}{\partial \sigma_{j}^{t}}\right\}
\end{aligned}
$$

The inverse of the derivative of the residual $\left[\frac{\partial R_{i}^{t}}{\partial \sigma_{j}^{t}}\right]^{-1}$ is used for the iterative stress correction within the subroutine loop (see figure 4) and the new trial stress increment is found from

$$
\Delta \sigma_{j}^{t, \text { trial }, \text { new }}=\Delta \sigma_{j}^{t, \text { trial }, \text { old }}-\left[\frac{\partial R_{i}^{t}}{\partial \sigma_{j}^{t}}\right]^{-1} R_{i}^{t}
$$

Once the iterative algorithm has converged ABAQUS requires an update of the Jacobian matrix that is obtained from

$$
\frac{\partial \sigma_{j}^{t}}{\partial \varepsilon_{i}^{t}}=\left[\frac{\partial R_{i}^{t}}{\partial \sigma_{j}^{t}}\right]^{-1}
$$

\section{Verification of UMAT Subroutine}

The accuracy of the nonlinear viscoelastic model implemented in the ABAQUS UMAT subroutine was verified against analytical solutions and experimental creep strain measurements on four pressurized cylinders.

\section{A. Experiment}

The cylinders had a diameter of $300 \mathrm{~mm}$ and height of $760 \mathrm{~mm}$, and were made of SF430. The overall test layout can be seen in Figure 5(a).

\section{Specimen Preparation and Experimental Setup}

Two layers of SF430 were placed on top of each other and heat-sealed with a soldering iron. Symmetry was achieved by forming two diametrically opposite, parallel seams. End-fittings made of MDF panels were attached to the cylinder with jubilee clamps and sealed with silicone and felt padding.

At mid-height the cylinder was fitted with nine self-adhesive coded targets for photogrammetry strain measurements, plus 2 targets for reference. The targets were located over a $40 \times 40 \mathrm{~mm}$ square region, see Figure 5(b).

The bottom end fitting was clamped to a rigid support, while the top end fitting was suspended from a string going over a pulley arrangement to a counterweight hanger, see Figure 5(a). The counterweight 


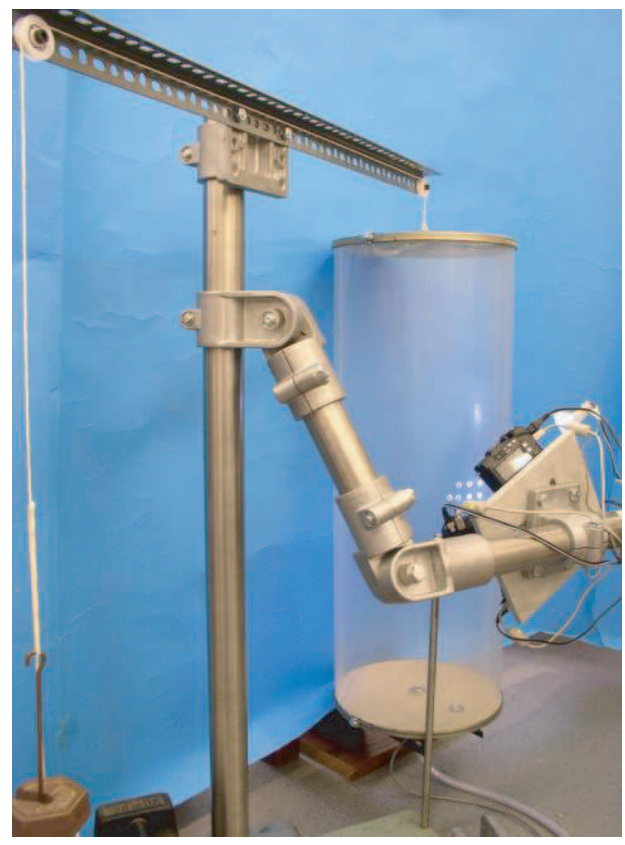

(a) Experimental setup

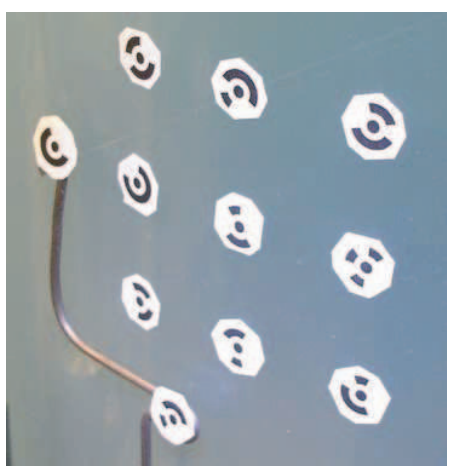

(b) Measurement region

Figure 5. Cylinder specimen.

hanger was equipped with a load transducer to allow for axial loads to be recorded. The cylinder was attached to an airline for pressurization and to a Sensor Technics pressure transducer with a maximum pressure of $2500 \mathrm{~Pa}$.

Four Olympus SP-350 digital cameras (with a resolution of $8.0 \mathrm{Mpixel)}$ were connected to a personal computer and mounted in front of the target area. The cameras were triggered via the computer and so produced sets of four simultaneous photos of the coded targets, each from a different view point.

\section{Procedure}

Table 2. Cylinder experiments

\begin{tabular}{|c|c|c|c|c|c|}
\hline Cylinder & Pressure $[\mathrm{Pa}]$ & Axial load $[\mathrm{N}]$ & $\begin{array}{c}\text { Material } \\
\text { orientation }\end{array}$ & $\begin{array}{c}\text { pressurised } \\
\text { after }[\mathrm{s}]\end{array}$ & $\begin{array}{c}\text { axial load } \\
\text { applied at }[\mathrm{s}]\end{array}$ \\
\hline 1 & 1100 & 22.22 & $\mathrm{MD}=$ hoop & 10 & 1 \\
\hline 2 & 1450 & 1.96 & $\mathrm{MD}=$ hoop & 60 & 35 \\
\hline 3 & 1000 & 29.92 & $\mathrm{MD}=$ axial & 4 & 5 \\
\hline 4 & 1450 & 1.96 & $\mathrm{MD}=$ axial & 60 & 35 \\
\hline
\end{tabular}

Initially, the weights on the counterweight hanger were set to match the weight of the top end fitting, in order to remove any load from the cylinder. The pressure regulator was set to the nominal experimental pressure, see Table 2. At the same time the axial load on the cylinder was increased by hanging additional weights through the counterweight hanger. Cylinder pressure and additional axial loads were recorded throughout the experiment. While the increase of the counterweight resulted in an instantaneous increment in axial loading, the pressure was applied in a ramp function and full pressurisation was reached only after several seconds. Table 2 provides details about the individual loading sequences.

Under loading the viscoelastic film deformed and the movement of the coded targets was recorded by taking a series of close-up photos. Photos of the measurement area were taken, initially at intervals of a few seconds and increasing to five minutes towards the end of the experiment. Three-dimensional coordinates of the targets were then computed by means of the photogrammetry software Photomodeler Pro5. The strain components in the axial and hoop directions were computed from the changes of coordinates of the targets over time. 


\section{B. Analytical Verification}

In terms of stress distribution the cylinder models can be regarded as "simple structures". Hence, neglecting the narrow regions close to the end-fittings, a uniform biaxial state of stress can be assumed throughout the structure. Further, the thickness of the film was assumed to remain constant over time. Once the loading of the cylinder is known over time the stresses in axial and hoop direction can be computed from

$$
\begin{gathered}
\sigma_{\text {hoop }}=\frac{r p}{T} \\
\sigma_{\text {axial }}=\frac{r p}{2 t}+\frac{F_{\text {axial }}}{2 r \pi T}
\end{gathered}
$$

Pressures and axial loads were recorded during the experiments. Based on these records it was possible to describe the loading of the structure over time with a series of ramp and step functions. Figure 6(a-d) shows plots of the resulting variation with time of the stresses in the machine and transverse material directions, for each cylinder. Using these stress functions and the viscoelastic material model from section III, together with equation 8 , the variation with time of the strains was computed analytically. The results are plotted together with the numerical results from ABAQUS, for comparison, in figures 710 .

\section{ABAQUS Analysis}

It was assumed that the cylinder is under a biaxial state of stress throughout the experiment, and so wrinkle free. This allows the film to be modelled with triangular membrane elements; a mesh of 72 M3D3 elements in the hoop direction and 56 elements in the longitudinal direction was used. Wrinkling of the film, which occurs near the end-fittings, was neglected because the creep measurements were taken away from the ends. One end fitting was fully constrained, whereas the other end of the cylinder was allowed to move axially.

A uniform internal pressure was applied, through the DLOAD command, and axial forces were applied through the CLOAD command. The variation with time of these loads was such that the resulting axial and hoop stresses were following the functions of time plotted in figures $6(\mathrm{a}-\mathrm{d})$.

The film was assumed to be anisotropic with material properties as in section III and the thickness was set to $0.038 \mathrm{~mm}$. The analysis was run at a constant room temperature of $23{ }^{\circ} \mathrm{C}$.

\section{Comparison of Results}

Four cylinders were tested at different pressures and with different axial loads, see Table 2. The first two structures were built with the material's machine direction (MD) aligned with the cylinder's hoop direction, whereas the remaining two had the machine direction aligned with the cylinder axis. The nominal hoop and axial stresses (calculated from Equations 14 and 15) are listed in table 3 together with the ratio between the stress in the machine direction and the stress in the transverse direction.

Table 3. Nominal biaxial stress states

\begin{tabular}{|c|c|c|c|}
\hline Cylinder & Hoop stress & Axial stress & $\begin{array}{c}\text { stress ratio } \\
\sigma_{M D} / \sigma_{T D}\end{array}$ \\
\hline 1 & 4.34 & 2.79 & 1.56 \\
\hline 2 & 5.72 & 2.92 & 1.96 \\
\hline 3 & 3.95 & 2.81 & 0.71 \\
\hline 4 & 5.72 & 2.92 & 0.51 \\
\hline
\end{tabular}

However during the experiments the nominal stresses were reached only after an initial loading period of 10-60 seconds. The detailed loading sequence shown in figures $6(\mathrm{a}-\mathrm{d})$ was derived by recording axial load and pressure and making use of Equations 14 and 15. Cylinder 1 was loaded axially at the time when the pressurisation process had just started, which is the reason for an initially higher stress in the transverse material direction. Cylinder 2 was pressurised slowly over 60 seconds and only a very small axial load was applied after about 35 seconds. The same experiment was repeated with cylinder 4 , but with material directions turned by $90^{\circ}$. For cylinder 3 , at the beginning of the experiment the pressure overshot and the nominal pressure was reached only at $\mathrm{t}=10 \mathrm{~s}$. The stress plots enabled us to consider the correct state of stress in both the finite elements model and the analytical verification. 


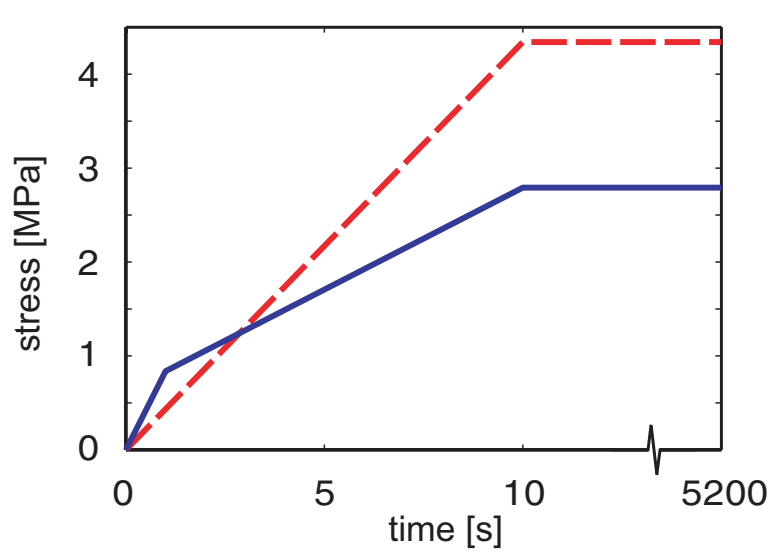

(a) Stress over time applied to cylinder 1

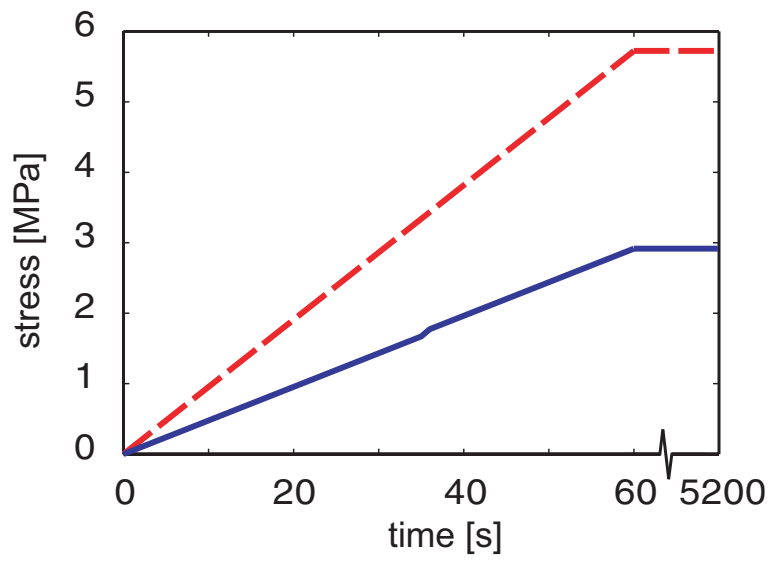

(b) Stress over time applied to cylinder 2

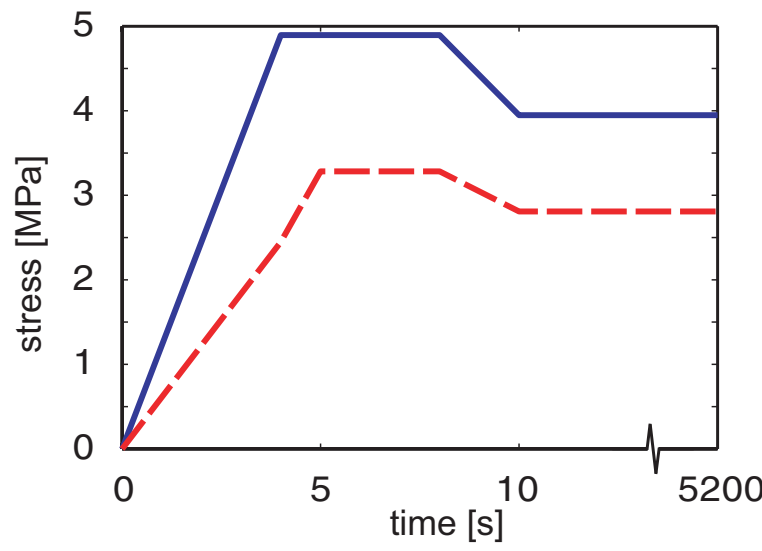

(c) Stress over time applied to cylinder 3

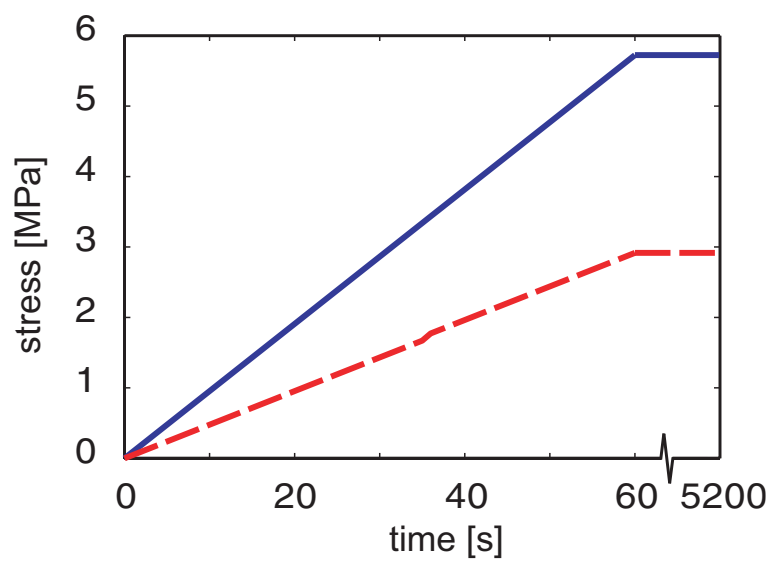

(d) Stress over time applied to cylinder 4

Figure 6. Stresses applied over time 
The test results from the experiments and the finite element analysis with ABAQUS have been plotted together with the analytical results in figures 7-10. Note that in these figures the total axial and hoop strains from ABAQUS are compared to the analytical results over logarithmic time, to allow for detailed observation especially at shorter times, whereas the experimental results are shown in comparison to the ABAQUS results over linear time, since the photogrammetric measurement method allowed only measurements to be taken in increments of 5 seconds, starting from $t=1 \mathrm{~s}$.

The comparison of results between ABAQUS and the analytical solution shows very good agreement and proves that the implemented subroutine works correctly. For cylinders 1 and 2, the ABAQUS and analytical results model practically coincide, while for cylinders 3 and 4 there are minor deviations. These may be due to local numerical instabilities or the need to refine the time increments and still needs to be further investigated.

Comparison of the experimental with the results from ABAQUS shows that the simulation works better for some stress states and less well for others. With cylinders 1 and 2 small discrepancies are observed (up to $10 \%$ at $\mathrm{t}=5000 \mathrm{~s}$ ) but in general the finite element simulation predicts the correct viscoelastic behaviour. However the experimental results from cylinders 3 and 4 show significant differences; in both cases the predictions for the hoop direction strains were about $20 \%$ lower, after $5000 \mathrm{~s}$, than the experimental observations. The predicted axial strains in cylinder 3 were about double the values observed and cylinder 4 showed decreasing axial strains (-0.7\% after $5000 \mathrm{~s})$ while from the simulation the strains were expected to remain approximately zero.

\section{Conclusion}

A numerical algorithm has been presented for the multiaxial formulation of Schapery's nonlinear viscoelastic material model. The iterative algorithm has been implemented for displacement based finite element programs using the user-defined-subroutine interface available in ABAQUS. Using the viscoelastic material data from StratoFilm 372, published by Rand and co-workers, the numerical model has been verified analytically and experimentally by means of cylindrical balloon structures.

Different stress ratios $\sigma_{M D} / \sigma_{T D}$ were applied to four cylindrical specimens, assuming a uniform stress distribution. Analytical and numerical results show good agreement and verify that the numerical implementation works well. Experimental results agree with the numerical results for high $\sigma_{M D} / \sigma_{T D}$ ratios, whereas agreement is less good for more balanced states of stress, i.e. when the stress applied in the transverse material direction is higher. In particular this becomes apparent in the last two cylinder experiments. An increase of S22 (i.e. less anisotropy) and S12 (i.e. higher Poisson's ratio) might describe the experimental observations more closely.

Finally, it should be noted that in this paper only uniform stress states were investigated in detail. While the model describes the viscoelastic material behaviour well for some stress states, significant differences were observed for others. More work needs to be done to evaluate this model on more complex states of stress.

\section{Acknowledgments}

We thank Dr Jim Rand for continuing advice and for providing film material data to us, and Dr David Wakefield for insightful suggestions. Mr Danny Ball (Columbia Scientific Balloon Facility), and Mr Loren Seely (Aerostar International) have provided advice and materials for this study. Financial support from the NASA Balloon Program Office is gratefully acknowledged.

\section{References} 2004.

${ }^{1}$ CAthey, H.M. Test flights of the NASA ultra-long duration balloon. Advances in Space Research, 33, 1633-1641,

${ }^{2}$ Gerngross, T., Xu, Y., And Pellegrino, S. Viscoelastic Behaviour of Pumpkin Balloons. To appear in Advances in Space Research, 2007.

${ }^{3}$ WAKEField, D. Numerical modeling of pumpkin balloon instability. AIAA 5th Aviation, Technology, Integration and Operations Conference, AIAA-2005-7445, 2005.

${ }^{4}$ Pagitz, M., AND Pellegrino, S. Computation of buckling pressure of pumpkin balloons. 47th AIAA/ASME/ASCE/AHS/ASC Structures, Structural Dynamics and Materials Conference, AIAA-2006-1803.

${ }^{5}$ Henderson, J.K., Caldron, G., And Rand, J.L. A nonlinear viscoelastic constitutive model for balloon films. AIAA 32nd Aerospace Sciences Meeting and Exhibit, AIAA-1994-638, 1994.

${ }^{6}$ Rand, J.L., Grant, D.A., And Strganac, T. The nonlinear biaxial characterization of balloon film. 34th AIAA Aerospace Sciences Meeting and Exhibit, AIAA-96-0574, 1996. 


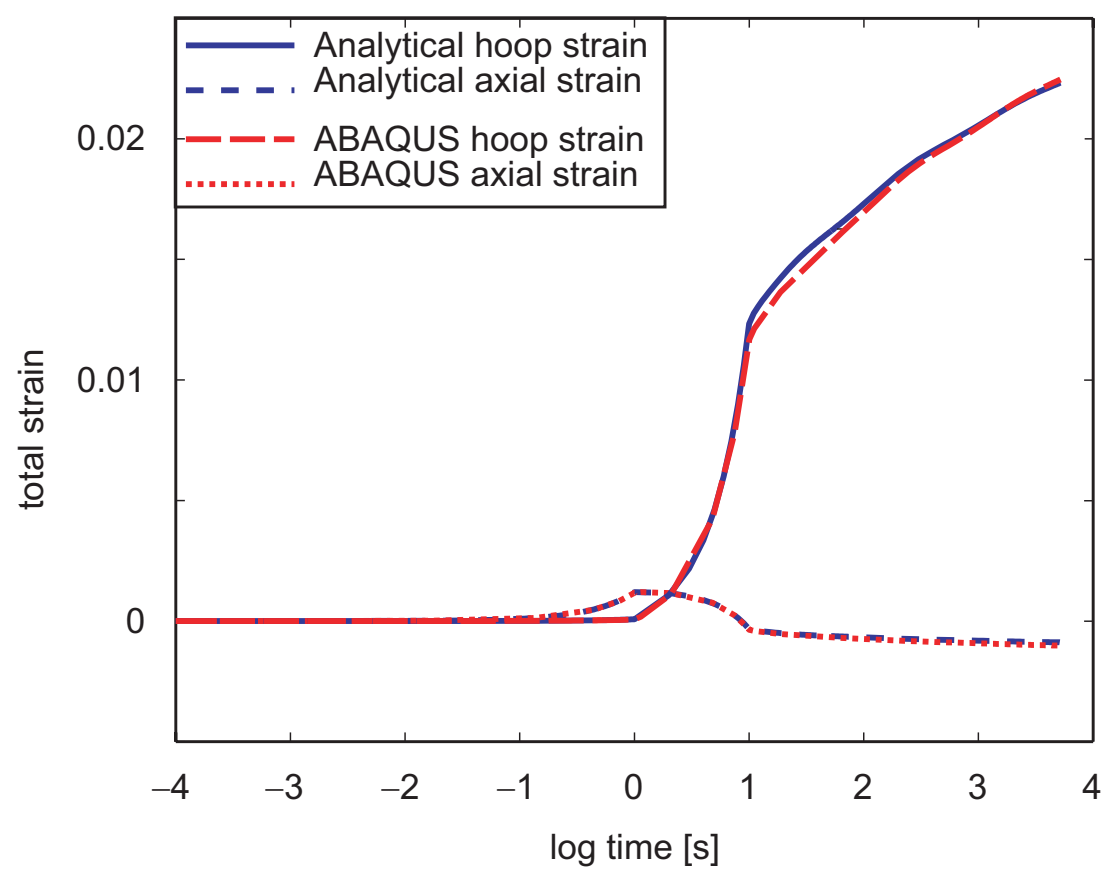

(a) Analytical and ABAQUS strains

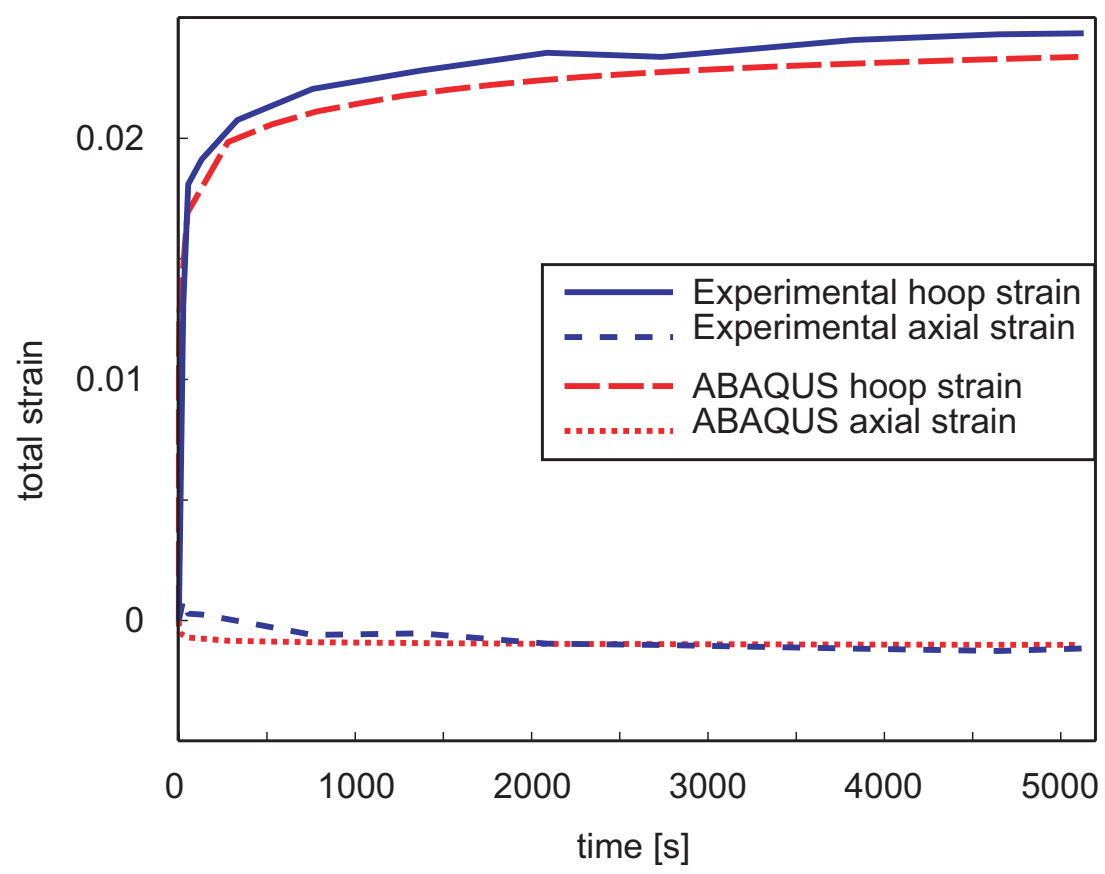

(b) Experimental and ABAQUS strains

Figure 7. Comparison of results for cylinder 1 


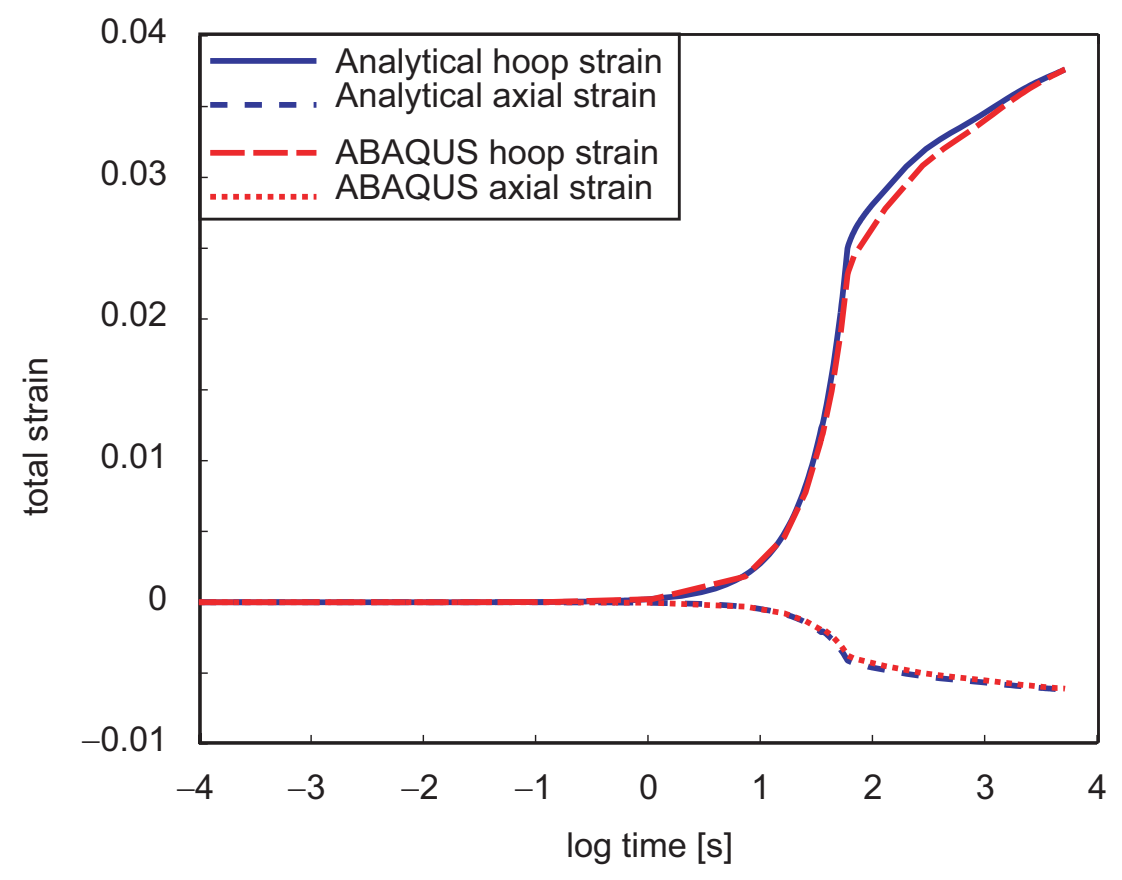

(a) Analytical and ABAQUS strains

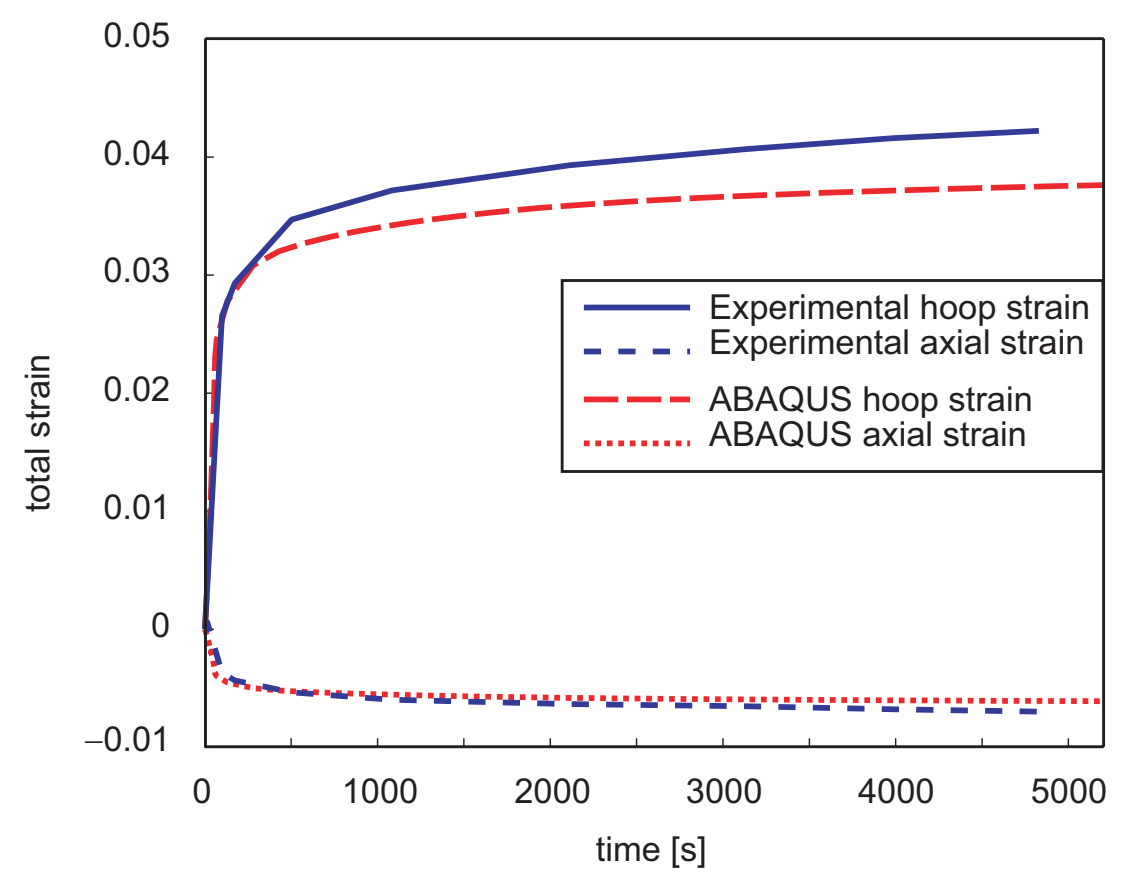

(b) Experimental and ABAQUS strains

Figure 8. Comparison of results for cylinder 2 


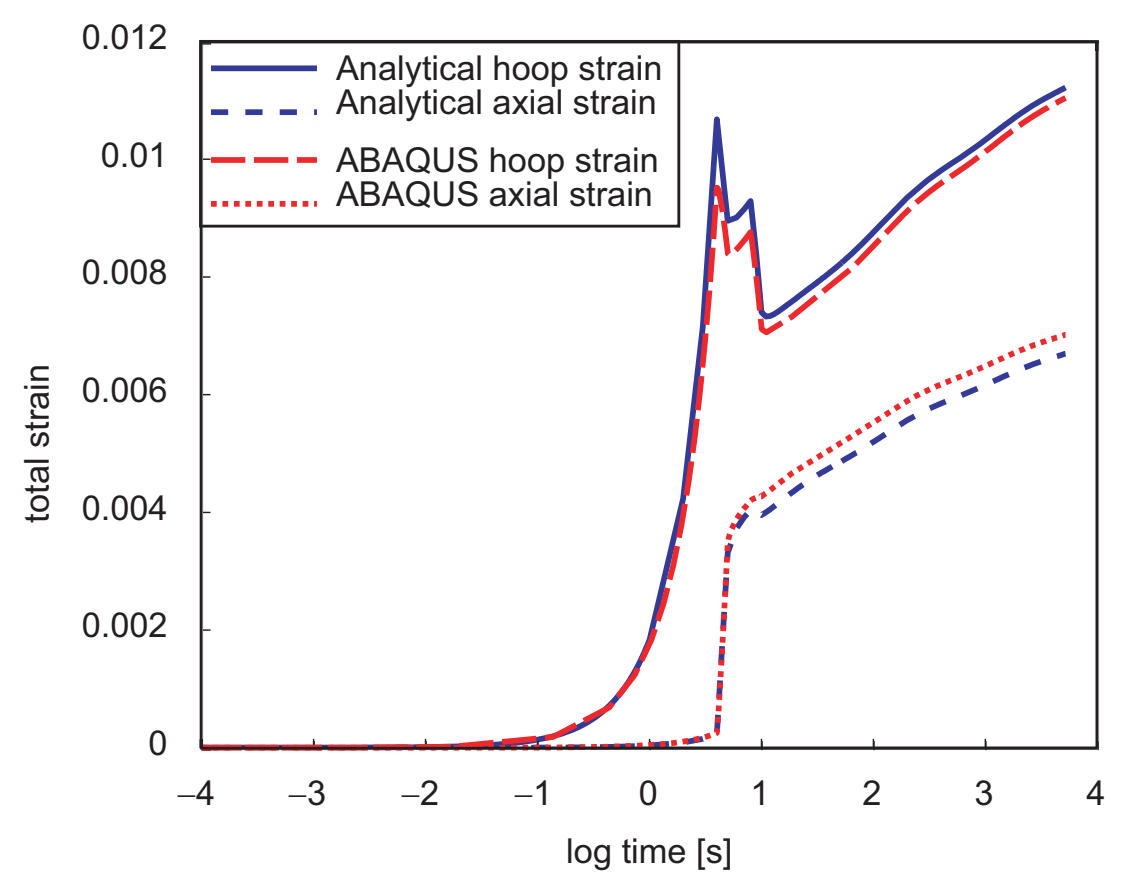

(a) Analytical and ABAQUS strains

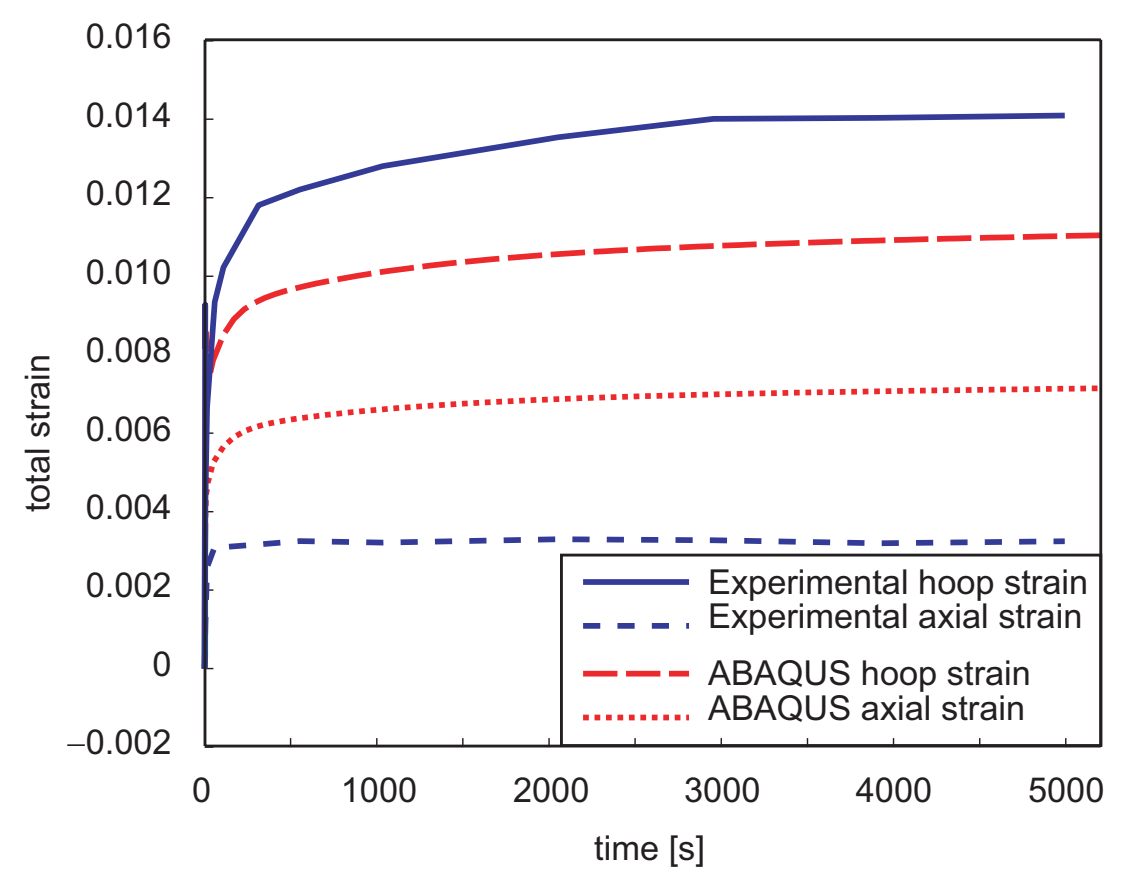

(b) Experimental and ABAQUS strains

Figure 9. Comparison of results for cylinder 3 


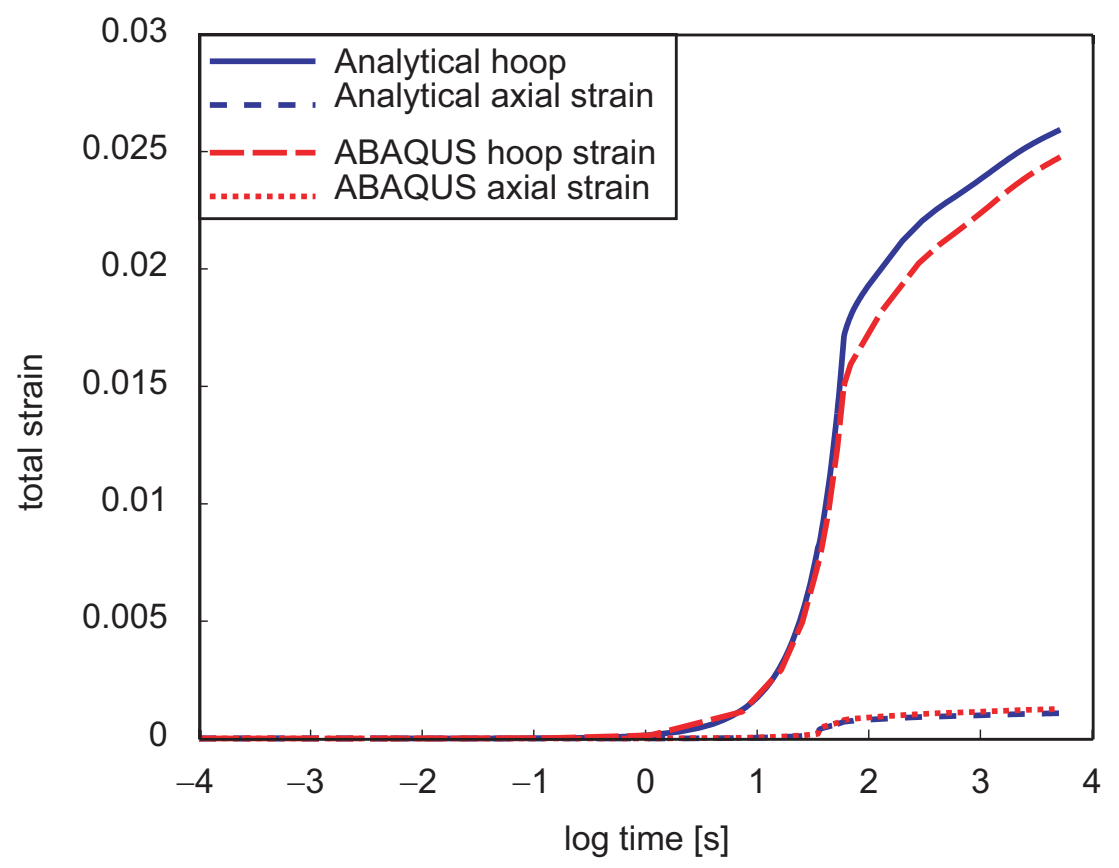

(a) Analytical and ABAQUS strains

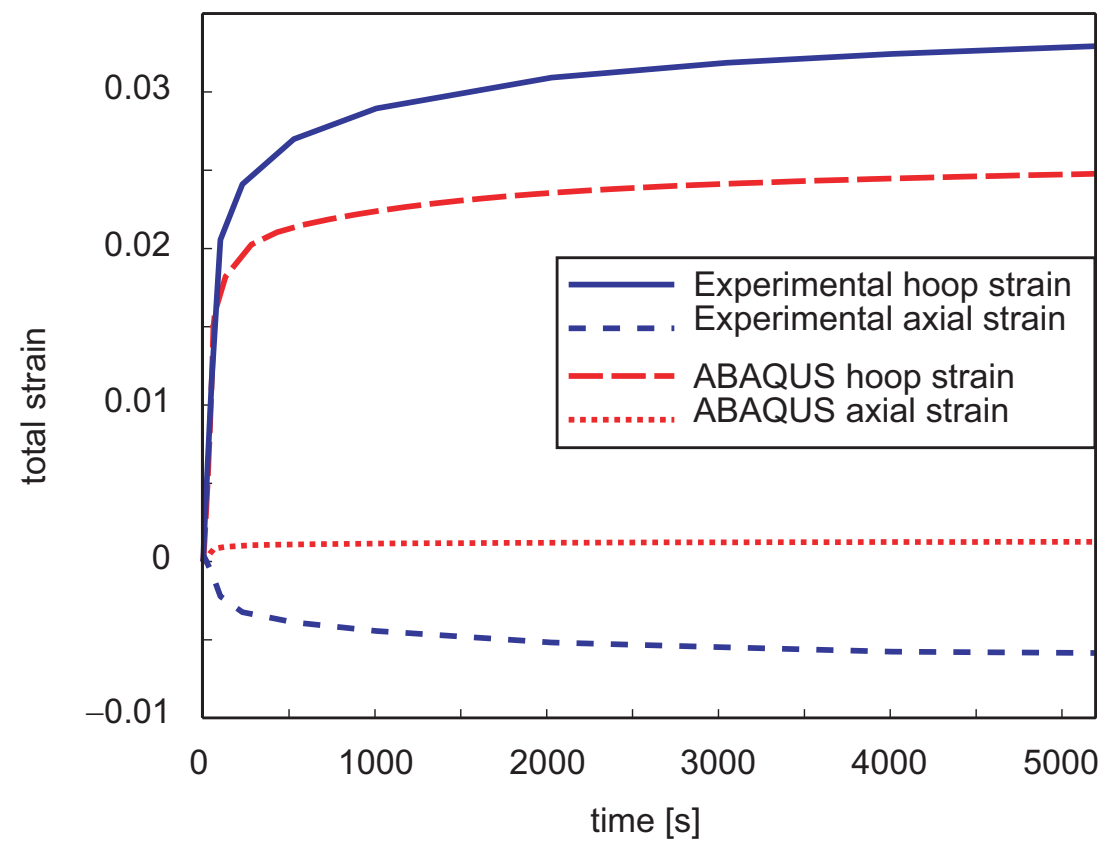

(b) Experimental and ABAQUS strains

Figure 10. Comparison of results for cylinder 4 
${ }^{7}$ Rand, J.L., Henderson, J.K., And Grant, D.A. Nonlinear behaviour of linear low-density polyethylene. Polymer Engineering and Science, 36, 8, 1058-1064, 1996.

${ }^{8}$ McCrum, N.G., Buckley, C.P., And Bucknall, C.B. Principles of Polymer Engineering, 2nd edition ed. Oxford Science Publications, Oxford, 2003.

${ }^{9}$ Ward, I.M. Mechanical Properties of Solid Polymers, 2nd edition. John Wiley \& Sons, 1985.

${ }^{10}$ Schapery, R.A. On the characterization of nonlinear viscoelastic materials. Polyner Engineering and Science, 9, 4, 1969

${ }^{11}$ Schapery, R.A. Nonlinear viscoelastic and viscoplastic constitutive equations based on themodynamics. Mechanics of Time-Dependent Materials, 1, 209-240, 1997.

${ }^{12}$ Rand, J.L., And Sterling, W.J. A constitutive equation for stratospheric balloon materials. Advances in Space Research, 37, 11, 2087-2091, 2006.

${ }^{13}$ Haj-Ali, R.M., and Muliana, A.H. Numerical finite element formulation of the schapery nonlinear viscoelastic material model. International Journal for Numerical Methods in Engineering, 59, 25-45, 2004.

${ }^{14}$ Sterling, And RAnd. Biaxial stress limit for ULDB film. AIAA 5th Aviation, Technology, Integration and Operations Conference (ATIO), AIAA-2005-7470, 2005. 